\title{
IV. NATION UND FORTSCHRITT
}

In den öffentlichen Denkmälern, die in den Kapitalen bis in die 1870er Jahre hinein entstanden, spiegelte sich wider, daß nationale Sinnkonstruktionen nicht nur auf die eigene Geschichte, die bestehende Staatsordnung und die militärische Leistungsbilanz oder Tradition zielten. Unter dem Eindruck der modernen Forschungs- und Wissenschaftsrevolution des 19. Jahrhunderts bildete daneben auch die Idee des Fortschritts eine Größe, zu der sich die Verfechter bestimmter Nationsbilder ins Verhältnis zu setzen suchten. Während diese Deutungsvalenz ab den 1870er Jahren zu einem zentralen Thema öffentlicher Denkmalsetzungen in den Hauptstädten avancieren konnte, zeichnete sich eine entsprechende Tendenz in der Zeit nach der Jahrhundertmitte zunächst erst allmählich und gemessen an der überragenden nationalsymbolischen Präsenz von Monarchie und Militär mit deutlich nachrangigem Stellenwert ab. Neue Impulse und Facetten hat das öffentlich inszenierte nationale Selbstbild gleichwohl auch in dieser frühen Phase erhalten.

\section{Paris: (Niederlagen-)Kompensation und politische Überformung}

Die kurze Dauer der Zweiten Republik erschwerte nicht nur die Fixierung eines Staatssymbols, sie ließ einer öffentlichen Denkmalpolitik generell kaum Spielraum. Noch bevor die napoleonische Repräsentationspolitik einsetzen konnte, kam - eher von der Zweiten Republik geduldet denn betrieben - vor dem Coup d'État im Dezember 1851 nur noch ein Denkmal für den Militärarzt Napoleons I., den medizinischen Forscher und Leiter des ehemaligen Militärhospitals Val-de-Grâce, Dominique Larrey, zustande. Maßgeblich vom Militär initiiert und finanziert, wurde es am 8. August 1850 im dortigen Innenhof südwestlich vom Jardin du Luxembourg im 5. Arrondissement eingeweiht, und bereits die Festszene ließ hier eine massive politisch-reaktionäre Überformung der Veranstaltung deutlich erkennen. Bei der nachmittäglichen Enthüllungszeremonie erhielten der Präsident der Assemblée nationale Dupin, der Komiteepräsident und General Petit sowie weitere Generale und Kommandanten der Nationalgarde Ehrenplätze zu Füßen des Denkmals. Sonst befanden sich auf den zu Sitzplätzen umfunktionierten Treppenstufen der Kirche von Val-deGrâce zahlreiche uniformierte Mitglieder des Medizinischen Corps und auf den mit Fahnen geschmückten Tribünen Mitglieder der seit der Verfassungsgebung vom November 1848 institutionalisierten Assemblée législative, des 
Institut de France und mehrerer Akademien aus Paris. Dazu kamen die Geistlichen des Val-de-Grâce sowie eine Abordnung von Invaliden und Veteranen in den Uniformen der napoleonischen Armée impériale neben nicht näher genannten Vertretern der »élite de la société parisienne « ${ }^{1}$. Bereits die starke Präsenz von Militärs und Veteranen rückte die Denkmalfeier deutlich näher an das Empire als an die Festpraxis der Republik, die in der Tat im Spätsommer 1850 bereits von der konservativen Transformation seit der Präsidentschaft des Bonaparte-Neffen Louis Napoléon erfaßt war.

Die Redebeiträge variierten das seit dem Subskriptionsaufruf vom Dezember 1842 verlautbarte Motiv und koppelten die Themen von medizinischem Fortschritt und Krieg eng aneinander: Larrey galt als "symbole d'humanité et de réparation sur presque tous les champs de bataille de la République et de l'Empire ${ }^{2}$. Seinem altruistischen »héroïsme« verdanke fast jede französische Familie die Rettung eines in den napoleonischen Kriegen des ausgehenden 18. und frühen 19. Jahrhunderts kämpfenden Mitglieds. Auch wenn Larreys "Mission« neben der "Patrie« noch der »science« gegolten hatte, war sie immer vorrangig dem »soldat « zugute gekommen ${ }^{3}$. Zum einen bildeten damit unversehens die napoleonischen Kriege, freilich abgesehen von der Niederlage gegen die Alliierten, den Inszenierungshintergrund für die Larrey-Statue. Damit wurden zugleich kompensatorische Erinnerungsstrategien gewählt, indem die Kriege als prägendes Kollektivereignis thematisiert wurden, ohne das Untergangsszenario zu erwähnen, das wenige Jahre später 1853 im Zuge der Feier für Ney so betont werden sollte ${ }^{4}$. Zum anderen fügten sich medizinische Wissenschaft, Patriotismus und die französische Armee zu einer Wertetrias, die es erlaubte, Differenzen zwischen Republik und Empire mit Blick auf die sie beide überwölbende »France« und deren Soldaten konsensual zu übergehen.

Die Festansprachen 1850 hielten sich lange mit den Karrierestationen Larreys auf, betonten die von ihm angeregte Modernisierung von Operationsmethoden und medizinischem Gerät ebenso wie seine theoretische und pädagogi-

1 Vgl. [An.,] Compte rendu des travaux de la commission de souscription pour le monument de Larrey érigé au Val-de-Grâce, le 8 août 1850, Paris 1850 (suivi de treize discours prononcés à l'inauguration du monument), S. $10 \mathrm{f}$. Die gegenüber der »assemblée importante« abqualifizierte "population « konnte nur von der Straße aus versuchen, die Zeremonie mitzuverfolgen. Vgl. ibid. und Le Constitutionnel, 9. August 1850, S. 2. Militärmusik spielte, bis die Hülle um das Denkmal unter Fanfarenklängen und dem Applaus der Festgesellschaft fiel und eine ganze Serie von Ansprachen involvierter Kommissions- und sociétéPräsidenten folgte. Vgl. [An.,] Compte rendu, s.o., S. 1lf.

2 [An.,] Souscription pour un monument à élever à la mémoire du Baron Larrey, Paris (14 décembre) 1842, S. 2.

$3 \mathrm{Vgl}$, ibid.

4 Vgl. dazu bereits Kapitel III. 1 im Ersten Teil. 
sche Forscherleistung an den renommierten Pariser Instituten ${ }^{5}$. Dabei galt Larreys Ausnahmeleistung immer auch als Indiz für die Privilegierung und providentielle Sonderstellung der französischen Nation, der es beschieden war, geniale Persönlichkeiten zu Zeiten der Bedrängnis hervorzubringen ${ }^{6}$. Leistungsvermögen und individuelle Selbstaufgabe zugunsten des Landes wurden damit zum eigentlichen Kraftreservoir der Nation.

Nicht nur in der Rede des Generals Petit, der sich namens der Armee an die Versammlung wandte, erschien Larrey gleich in vierfacher Hinsicht als konsensuale Figur: zunächst jenseits der politisch gegensätzlichen Regime von Erster Republik, Empire und Restauration, dann jenseits der Differenzierung in militärische und zivile Welt, weiter selbst jenseits der territorialen Grenzen Frankreichs ${ }^{7}$ und zuletzt noch jenseits aller sozialen und Ranghierarchien ${ }^{8}$. Darüber hinaus zeugte die Feier ihren Zelebranten zufolge davon, daß Frankreich längst nicht mehr nur seinen Militärs, sondern auch seinen Bürgern Denkmäler setzte: »si trop longtemps la patrie n'avait voulu couronner que des soldats victorieux, elle (...) consacre [aujourd'hui] des monuments à ceux qui ne se sont imposés d'autre mission que celle de conserver des citoyens: monuments pieux «". Mitunter beflügelte dies sogar den Wunsch nach einem "Panthéon médical«, das Frankreich seinen renommierten Vertretern der "sciences médicales« widmen sollte ${ }^{10}$.

Darüber hinaus betonten die Redner eindringlich, daß Napoleon Bonaparte es gewesen sei, der Larrey auszeichnete ${ }^{11}$, wie sich überhaupt die Tatsache, daß Larrey an den großen militärischen Expeditionen Frankreichs teilgenommen und die französischen Siege durch die Versorgung der Verletzten mit habe ermöglichen können, maßgeblich napoleonischer Patronage verdanke. Spätestens wenn Napoleon dann in panegyrischem Ton als »celui qui (...) dominait (...) par génie ${ }^{12}$ umschrieben wurde, mündete der Larrey- direkt in den Napoleon-Kult. Der Vertreter der Académie des sciences, Roux, ergänzte eine

5 Vgl. Discours prononcé par M. le Général Petit, in: [An.,] Compte rendu, S. 14-17, hier S. 16.

6 Discours prononcé par M. Bégin, in: ibid. S. 13 und 18-30 [durch Fehldruck], hier S. 18: "C'est un des précieux privilèges de ce pays, que de produire presque à point nommé les hommes dont il a le plus besoin. (...). Notre histoire est glorieusement remplie des témoignages de cette fécondité providentielle, qui jamais, il faut l'espérer, ne lui fera défaut. « So auch M. Roux, ibid. S. 31-42, hier S. 31.

7 Discours prononcé par M. le Général Petit, ibid. S. 17: »Il continua à servir sous la République, sous l'Empire (...). Dignement apprecié et aimé des gens de guerre (...), il était aussi estimé et honoré des premiers personnages de l'État et des sociétés savantes (...).«

8 Vgl. ibid. S. 15.

9 Discours prononcé par M. Dubois (au nom de l'Académie nationale de médecine), in: ibid. S. 43-49, hier S. 49 .

${ }^{10}$ Vgl. Disocurs prononcé par M. Roux, ibid. S. 34

1 Discours prononcé par M. le Général Petit, ibid. S. 14.

12 So der Vertreter der Subskriptionskommission, Bégin, in seinem Discours, ibid. S. 20. 
neue Pointe, indem er nicht nur Larreys Verdienst für die Nation von Napoleon Bonaparte her berechnete, sondern Larreys Denkmalehrung 1850 weniger der Zweiten Republik als direkt ihrem mächtigen Präsidenten zuerkannte, dessen Autoritätsschub an der Spitze der "patrie« er knapp ein Jahr vor dem Staatsstreich antizipierte:

Trente ans se sont écoulés depuis la mort de l'empereur; et, après cette période de temps, marquée par tant de caprices de la fortune, par tant d'étranges volontés de la Providence, un monument est élevé à la mémoire de Larrey par la France de nouveau constituée en république, et au moment où la première magistrature de l'État est confiée à un neveu de Napoléon (...), celui qui tient dans ses mains peut-être les destinées de notre patrie ${ }^{13}$.

$\mathrm{DaB}$ eine nennenswerte Nationalgeschichte nicht früher als mit Napoleon Bonaparte begann und die Zukunft der Nation in einer Art napoleonischen Kontinuitätsstiftung würde liegen können, war die konservative Vision und Prognose, die Roux den Zuhörern hier anzubieten hatte.

Während die republikanische Regierung mit dem Wettbewerb um die Figure de la République von 1848 versucht hatte, über die Allegorie zu einer Definition der französischen Nation zu gelangen, die im Kern auf die neue republikanische Ordnung zielte ${ }^{14}$, wurde diese in den offiziellen Deutungen des Larrey-Denkmals gezielt übergangen. Statt dessen bezog sich die Exempelfunktion des Militärarztes gerade auf seine überparteiliche Selbstaufgabe zugunsten einer "patrie«, die weder politisch noch sozial differenziert, sondern unspezifisch-homogen erschien. Eher war das Loyalitätspostulat ganz auf eine providentiell-mystisch vorgestellten $» F r a n c e$ « gerichtet. Zugleich lag den Stilisierungen als Reflex auf den Untergang der napoleonischen Herrschaft 1815 ein vager Niederlagentopos zugrunde, wie er später nach 1871 auch für die republikanischen Nationskonzepte grundlegend werden sollte, indem Larrey die progressive Potenz der Nation unter den Vorzeichen existentieller Bedrohung verkörperte und damit Anlaß bot, ihre Regenerationskräfte zu beschwören. Die Presse ließ dieses Ansinnen unkommentiert ${ }^{15}$. Als offizielles Organ würdigte nur der Moniteur Universel das Prozedere und lobte am Ende symbolträchtig, daß die Feierlichkeiten zu keinem Zeitpunkt eine Gefahr für »le plus grand ordre« dargestellt hätten ${ }^{16}$.

Dezidiert republikanische öffentliche Denkmäler sind danach bis 1851/52 in der Kapitale nicht mehr entstanden. Währenddessen kündigten sich Unterwanderungen der Symbolmacht durch das bonapartistische Empire umso deutlicher an. Unter diesen Umständen zählte der Umstand, daß die Place de la Bastille als traditionell revolutionärer Symbolort bewahrt werden konnte, bereits zu den wenigen kultischen Errungenschaften der Zweiten Republik. Das Füh-

13 Discours prononcé par M. Roux, ibid. S. 33.

$14 \mathrm{Vgl}$. dazu bereits ausführlich Teil I, Kapitel I.1.

15 Vgl. knapp Le Siècle vom 9. August 1850, S. 2; Le Constitutionnel, 9. August 1850, S. 2.

16 Vgl. Le Moniteur Universel, 9. August 1850, S. 2777. 
rungspersonal der Provisorischen Regierung trug dieser topographischen Logik bereits Rechnung, indem die Republikaner Lamartine und Arago an der Spitze eines festlichen Zuges, zu Füßen der Julisäule auf dem Bastilleplatz, die Republik proklamierten ${ }^{17}$. Zur Entfaltung einer exklusiven Programmatik, mit der die Einbindung der konservativen Opposition hätte versucht werden können, bot sich während der kurzlebigen Zweiten Republik allerdings keine Möglichkeit.

Sobald das Zweite Empire etabliert war, schienen Denkmalehrungen für Fortschritts-Repräsentanten nicht mehr ganz so demonstrativ an eine nationale Idee zurückgekoppelt werden zu müssen. 1866 konnte ein Bronzedenkmal für den Agronomen Antoine Parmentier an halböffentlichem Ort im Botanischen Garten der École de Pharmacie im 6. Arrondissement eingeweiht werden, und Mitte der 1870er Jahre folgte das während der frühen 1860er Jahre bereits mitgeplante Denkmal für den Chemiker und Mineralogen Nicolas Vauquelin am gleichen Standort ${ }^{18}$. Die Denkmalfähigkeit solchen Personals leitete man vor allem im Falle der Parmentier-Ehrung höchstens verhalten von nationalpolitischen Kriterien her ${ }^{19}$. Immerhin betonte der Subskriptionsaufruf zugunsten des Denkmals für Vauquelin, daß dessen Forschung und Lehre im Bereich der Mineralogie Entdeckungen ermöglichte, die mehr noch als der sfortune publique« dem "progrès de l'Industrie française« nützten ${ }^{20}$. Als Chemiker habe er so gewirkt »pour conserver à la France la suprématie dans l'industrie chimique ${ }^{21}$. Parallel zum Versuch, Vauquelin als um den naturwissenschaftlichen Fortschritt und die internationale Wettbewerbsfähigkeit der französischen Nation verdiente Figur darzustellen, appellierten die Stifter ausdrücklich nicht an einen engen Expertenkreis potentieller Subskribenten, sondern wandten sich an die weite Adressatengruppe derer, die dem »développement de l'esprit

17 Vgl. République Française. Grande fête nationale. Proclamation de la République aux pieds de la Colonne de Juillet. Revue de la Garde Nationale, o. O. o. J. [1848], S. 1. Freilich wiederholte sie in diesem Akt vom 27. Februar 1848 nurmehr die bereits drei Tage zuvor erfolgte Ausrufung der Republik vom Hôtel de Ville im Stadtzentrum. Ebenso fand am 4. März 1848 die Beisetzung der Opfer der Februarrevolution als den smartyrs de la victoire« und »apôtres de la liberté« unter der Julisäule statt. Zuvor hatte ein Gedenkgottesdienst in der mit schwarzem Trauerflor verkleideten Église de la Madeleine und von hier aus der Trauerzug nach dem Bastilleplatz stattgefunden. Vgl. République Française. 1848. Char triomphal et funèbre qui a transporté, sous la Colonne de Juillet, les héros morts pour la patrie, o. O. o. J. [1848].

18 Vgl. auch HARGRove, Les statues de Paris. La représentation, S. 100f.

19 Vgl. Schreiben des Komiteesekretärs Chatin und Professor der École de Pharmacie an den Innenminister, AN F ${ }^{\text {lc }}$ I 168 Dossier: Projet des décrets (...) autorisant l'érection de statues à Parmentier et à Vauquelin.

${ }^{20} \mathrm{Vgl}$. Comité pour l'érection, à Paris, d'une statue de bronze à Vauquelin, AN ibid

21 Comité pour l'érection d'une statue de bronze à Parmentier et à Vauquelin, 1. Februar 1869, AN F 4856 Dossier: Monument Parmentier et Vauquelin, cour de l'École de Pharmacie, 20 jan. 1869-18 nov. 1875. 
d'invention ${ }^{22}$ in Frankreich verpflichtet waren. Die unterschwellig gebliebene nationale Zurechnung der individuellen Leistung sollte hier offenkundig auch dazu dienen, zusätzliche Beitragszahler zu mobilisieren, ohne daß sie das Unterfangen thematisch klar dominiert hätte.

Ähnlich verhalten national überformt blieb auch ein um 1864 verwirklichtes Denkmalprojekt für den Naturforscher und Mineralogen Louis Daubenton im Jardin d'Acclimatation am Nordende des Bois de Boulogne im 16. Arrondissement. Der Subskriptionsaufruf von 1861 feierte mit überschwenglichen Topoi den Fackelträger der »sciences naturelles«, seine zoologische Forscherleistung als Werk geistiger Aufklärung ${ }^{23}$ und seine systematische Artenerfassung regelrecht als Erfüllung des nach der Genesis überlieferten göttlichen Auftrags zur Domestizierung der Schöpfung ${ }^{24}$. Gleichwohl blieb das Argument zentral, daß die verbesserte Agrarproduktion, zu der Daubenton habe beitragen können, ihm die "reconnaissance de l'État« eintragen müsse »auquel il a donné une nouvelle source de prospérité «s. Als Summe solcher Qualifikationen wurde schließlich die Monumentalisierung als Akt der »reconnaissance nationale ${ }^{26}$ beansprucht. Ein Interesse der Nation am Denkmal suggerierten die Stifter demnach gleichermaßen auf theoretisch-wissenschaftlicher, religiöser wie pragmatischer Ebene ${ }^{27}$.

Die Denkmäler für die Vertreter einer naturwissenschaftlichen Progrès-Idee blieben bemerkenswert, weil sie ungeachtet mäßiger öffentlicher Resonanz das Bild der Nation um Kriterien nun nicht länger machtpolitischer oder militärischer, sondern rational-technischer Effizienz erweiterten. Gleichwohl spiegelten sie zugleich auch die Taktik zeitgenössischer Denkmalkomitees wider, die sich die nationale Vokabel durchaus auch anempfinden konnten, um unter diesem Etikett Öffentlichkeit und Zuspruch für ihr Projekt herzustellen.

Comité pour l'érection, à Paris, d'une statue de bronze à Vauquelin, $\mathrm{AN} \mathrm{F}^{1 \mathrm{c}}$ I 168 Dossier: s.o.

23 Vgl. [Drouyn DE LHYS,] Société impériale zoologique d'acclimatation. Projet d'élever une statue à Daubenton. Rapport fait à la Société dans le séance du 3 mai 1861, par M. D. de L., Paris 1861, S. 3.

24 Vgl. ibid. S. $3 f$.

25 Ibid. S. 10.

26 Ibid. S. 5.

27 Ebenfalls mäßige öffentliche Aufmerksamkeit erregte ein Denkmalprojekt, das ähnlich der Statue für den Militärarzt Larrey 1857 dem Mediziner Xavier Bichat nahe der École de Médecine im 6. Arrondissement gewidmet wurde. Vgl. zum Fest am 16. Juli: Le Siècle, 18. Juli 1857, S. 3. 


\section{Berlin: Inklusive Inszenierungen} von agrarischem und gewerblichem Fortschritt

Neben den um 1855 vor der Neuen Wache errichteten und den restaurierten Generalsdenkmälern auf dem Wilhelmplatz erweiterten die Standbilder für die preußischen Wirtschaftsreformer Albrecht Thaer (1860) und Peter-Christian Beuth (1861) auf dem Schinkelplatz an der Bauakademie und vor dem Königlichen Palais (Kronprinzenpalais) das Figurenarsenal auch in Berlin um Repräsentanten eines zivilen, fortschrittlichen preußischen Staates. Damit erhielten erstmals nach der Revolution jetzt nicht militärisch profilierte, sondern als Exponenten einer modernisierten Landwirtschaft und der Frühindustrialisierung gefeierte Personen Denkmäler, weil sie den ökonomischen Aufwärtstrend seit Jahrhundertbeginn mit ermöglicht hätten.

Im Subskriptionsaufruf für das Thaer-Denkmal von 1843 bescheinigte der Präsident des von Thaer gegründeten Landesökonomiekollegiums Georg Philipp von Beckedorff, daß »die größere Hälfte der deutschen Bevölkerung namentlich der norddeutschen und vor allen anderen die preußische« Thaer »als einen ihrer größten Wohltäter ${ }^{28}$ ehren wolle. Angesichts der auch in anderen deutschen Staaten angelaufenen Projekte schürte der Aufruf Konkurrenzdruck, um Beitragszahler für die preußische Initiative zu mobilisieren. Der Denkmaleinweihung Anfang November 1860 auf dem Platz vor der Bauakademie blieb der schon seit Oktober 1858 nicht mehr regierungsfähige preußische König Friedrich Wilhelm IV. krankheitsbedingt fern, obgleich sich das Komitee im Vorfeld massiv um seine Teilnahme an den Feierlichkeiten bemüht hatte ${ }^{29}$ und seine Anwesenheit nicht zuletzt auch als symbolische Favorisierung des hauptstädtischen gegenüber den in Leipzig und Celle bereits errichteten Monumenten erachtet wurde ${ }^{30}$. Statt dessen war eine nicht nur regional, sondern auch sozial beachtlich breit rekrutierte und zivil geprägte Festversammlung zustandegekommen: Ihr gehörten zunächst zahlreiche preußische Minister, Generale, Botschafter und hohe Beamte ${ }^{31}$ und Abordnungen der städtischen Gremien

28

29

Rep. 89 Nr. 20894, Bl. 67.

Vgl. Schreiben an den König vom 2. Oktober 1860, GStA PK ibid. Bl. 68.

31 Vgl. [Th. FonTANE,] Denkmal Albrecht Thaer's zu Berlin nach dem Entwurfe von Chr. Rauch ausgeführt von H. Hagen. Mit Text von Th. FONTANE, Berlin o. J., S. 2. Vertreten waren neben Feldmarschall von Wrangel, dem Chef des Generalstabs der Armee von Moltke und dem Kommandanten von Berlin von Alvensleben auch die Staatsminister von Auerswald, der Minister für Handel, Gewerbe und öffentliche Arbeiten v.d. Heydt, Justizminister Simons, Finanzminister von Patow, der Minister für landwirtschaftliche Angelegenheiten Graf Pückler, als Minister der geistlichen Unterrichts- und MedizinalAngelegenheiten von Bethmann-Hollweg und Innenminister Graf Schwerin. 
sowie Vertreter der Akademien und der Universität in Amtstracht, durchaus aber auch Teilnehmer aus anderen deutschen Staaten an. Gemäß dem bei offentlichen Denkmalfesten üblichen Modus kamen dazu neben dem Steinmetzmeister zunächst nur der am Denkmalbau beteiligte "Ciseleur« und ein Gießer als unterbürgerliche Vertreter ${ }^{32}$. Ungewöhnlich geprägt war die Feier unterdessen durch die zahlreichen Mitglieder des von Thaer gegründeten "Landesökonomiekollegiums« aus landwirtschaftlichen Vereinen und halbstaatlichen Gesellschaften und durch die nach Provinzen angeordneten, fast ausschließlich aus lokalen Beamten und adligen Gutsbesitzern rekrutierten Vereinsvertreter ${ }^{33}$. Eine bemerkenswerte Ausnahme vom sonst dominant bürgerlichen bzw. adligen Profil der Festgesellschaft bildeten schließlich die "200 Arbeiter der landwirthschaftlichen Maschinenfabriken « mit ihren Fahnen und Emblemen und einem eigenen Corps, die sich im Anschluß an einen Festzug durch die Stadt auf dem Platz aufstellten ${ }^{34}$ und deren »Vorbeimarsch« am Denkmal den knapp halbstündigen Festakt beendete ${ }^{35}$.

In seiner Rede würdigte der Komiteevorsitzende und Abgeordnete des preuBischen Herrenhauses von Meding Thaer erst als "Begründer der wissenschaftlichen Auffassung des Landbaus« und »Beispiel gemeinnütziger Thätigkeit ${ }^{36}$, um dann aber den überwiegenden Teil der Rede darauf zu verwenden, diese Leistungsbilanz an "König«, "Vaterland« und "Gott « als bedingende und übergeordnete Größen rückzukoppeln, bis der Thaer- als Facette des Monarchenkults erschien. Die Effizienzsteigerung in der Landwirtschaft zählte jetzt zu den "zahlreichen Wohltaten, welche das preußische Vaterland der gesegneten Regierung Sr. Majestät des Königs Friedrich Wilhelm III. des Weisen und Gerechten verdank[e]«, indem der nämlich den aus dem Königreich Hannover stammenden Thaer nach Preußen berufen hatte ${ }^{37}$. Das individuelle Verdienst erschien nun sekundär gegenüber preußisch-nationaler Loyalitätsbezeugung, indem das von Thaer repräsentierte Erwirtschaften »materieller Güter« prinzipiell einer Tugendtrias von »felsenfeste[r] Königstreue, ächte[r] Vaterlandsliebe und wahre[r] Gottesfrucht» untergeordnet wurde. Indem man die individuelle Ehrung zugunsten preußisch-monarchischer Werte relativierte, sollten offenkundig Gegensätze beschwichtigt und die moderne, effizienz- und

${ }^{32}$ Vgl. FONTANE, Denkmal Thaer's zu Berlin, S. IVf.

${ }^{33}$ Vgl. ibid. S. Vf.

34 Ibid. S. 3. Als Vertreter landwirtschaftlicher Vereine außerhalb Preußens wurden im einzelnen genannt: je ein Vertreter des Bayerischen Handelsministeriums, des sächsischen Innenministeriums und Vertreter von Vereinen in Wiesbaden, Celle, Hannover, Lüneburg, Prag, Brünn, Dresden, Chemnitz, Hamburg, Eisenach, Köthen, Roslau, Kiel sowie aus Kurland und dem schwedischen Karlskrona. Insgesamt nahmen etwa 1.200 geladene Gäste teil.

35 Vgl. ibid. S. 4.

${ }^{36} \mathrm{Vgl}$. die gesamte Rede ibid. S. 3.

37 Vgl. ibid. 
gewinnorientierte Wirtschaftgesinnung, wie sie Thaer propagiert hatte, an jenes traditionale Verständnis des Landbaus als Dienst am Staat herangerückt werden, an dem die preußische Gutswirtschaft ungeachtet der neuen agrarkapitalistischen Praxis festhielt. Am Ende beharrte von Meding aber vor allem noch einmal auf der Denkmalwürdigkeit nicht-dynastischen und nicht militärischen Personals, weil so zutage trat, 》dass das Vaterland die Verdienste seiner Söhne um die Künste des Friedens nicht minder ehr[e], wie die Helden und Staatsmänner ${ }^{38}$. Als Exponent ziviler Fortschrittsleitung vertrat Thaer somit gleichberechtigt das leistungsstarke "preußische und deutsche Vaterland ${ }^{39}$.

Einwände gegen von Medings Rede waren im Berliner Kommunalblatt zu lesen. Hier wurde ausdrücklich bestritten, daß der »Landbau« dazu berufen sei, durch Loyalität zu Gott, König und Vaterland beispielgebend zu wirken: "Wir halten vielmehr dafür, das es a 11 e n Classen und Ständen geziemt, in jenen Tugenden mit einander zu wetteifern und daß das Staatswohl nur da zur höchsten Blüthe gelangt, wo jeder nach dem Ruhme strebt, Sieger in diesem Wettkampf zu bleiben ${ }^{40}$. Hatte bereits die Festszene verdeutlicht, daß im Zeichen des gefeierten (land)wirtschaftlichen Aufschwungs ein durchaus inklusives Gesellschaftsbild vermittelt werden sollte, indem man selbst Arbeitern die Teilnahme am Fest genehmigte, so wurde nun auch ein emanzipatorischer Einwand laut, wonach die Nation sich nicht nur auf Effizienzzugewinne aus dem agrarischen Sektor stützen konnte, sondern - so blieb allerdings nur vage angedeutet - in steigendem Maße auch von den gewerblich-industriellen Aufschwüngen und also auch von den Kräften der gesamten und eben nicht nur einer elitär-bürgerlichen Gesellschaft zehrte. Der politische Tenor von Medings Rede, der die preußische Nation auf göttliche und monarchische Autorität hin fixiert hatte, blieb unterdessen unwidersprochen.

Mit der Initiative für das zweite Berliner Denkmal zugunsten eines Exponenten des »Fortschritts « wurde solcher leisen Kritik an einer Überbewertung agrarischer Produktionsverdienste um die preußische Nation unmittelbar Rechnung getragen. Im Subskriptionsaufruf zum Denkmal für Christian Beuth vom Dezember 1853 wurden nicht nur dessen reformpolitische Maßnahmen als Mitglied der Gruppe um Hardenberg und nach 1817 in verschiedenen Ministerien und dem Staatsrat gewürdigt ${ }^{41}$. Besonders betonte der Aufruf darüber hinaus die Anstöße zur unternehmerischen Privatinitiative, die Beuth zunächst im Rahmen einer staatlichen Deputation vermittelt hatte, dann im Kontext des

39 Vgl. ibid

40 Communal-Blatt der Haupt- und Residenzstadt Berlin, $\mathrm{N}^{\circ} 20$ vom 11. November 1860 , S. 173 [H. i. 0.].

41 Aufruf des Central-Komitees zur Errichtung eines Monuments für den Wirklichen Geheimen Rath Beuth vom Dezember 1853, GStA PK I. HA Rep. 93 C Nr. 2363, Bl. 45 Rs und Bl. 46. 
als Selbstverwaltungskörperschaft 1821 etablierten Vereins zur Beförderung des Gewerbefleißes in Preußen, den Unternehmer und andere Reformer, Minister und schließlich selbst der König und der Thronfolger unterstützten ${ }^{42}$. GroBe Bedeutung wurde ebenso der Institutionalisierung fachspezifischer Ausbildung beigemessen, wie sie in Form des von Beuth initiierten Gewerbeinstituts in Berlin als dem Multiplikator eines modern ausgebildeten Unternehmertums und in Gestalt weiterer Gewerbeschulen in anderen preußischen Provinzen gegriffen hatte. Am Ende bedingten neben staatlichen "Maaßregeln der Gesetzgebung und Verwaltung", vor allem die halbstaatliche beziehungsweise private "Heranbildung eines vielseitig unterrichteten, zur Aneignung jedes Fortschritts befähigten, der eigenen Kraft sich bewußt gewordenen Gewerbestandes« Beuths shohe und bleibende Verdienste (...) um die vaterländische Gewerbethätigkeit ${ }^{43}$.

Auch der Einweihung des Beuth-Denkmals Mitte Mai 1861 ging zunächst ein Festumzug von Abordnungen der Handwerker, Innungen und Fabrikarbeiter mit Fahnen und Emblemen voraus, die vom Lustgarten her zum Festplatz vor der Bauakademie zogen, das Denkmal umrundeten und sich um den Platz aufstellten $^{44}$. Anders als anläßlich der Einweihung des Thaer-Denkmals beehrte diesmal auch der nach dem Tod seines Bruders seit Beginn des Jahres regierende preußische König Wilhelm I. die knapp einstündige Feier zumindest mit distanzierter Präsenz, indem er von den geöffneten Fenstern der "Commandantur" im Zeughaus aus der Zeremonie folgte ${ }^{45}$. Zugleich erzwang der König damit die Ausrichtung des abschließenden Rituals - des Abzugs der Schüler des Gewerbeinstituts und der Bauakademie zum einen, der Handwerker und Innungen zum anderen - auf das Areal monarchischer und militärischer Repräsentation hin: Die Festzüge passierten nicht nur salutierend das Denkmal selbst, sie zogen auch mit der Ehrfurchtsgeste gesenkter Fahnen und entblößter Häupter am Monarchen und am Zeughaus vorbei. Mit dem Denkmal für Beuth mochte so das zweite nicht-dynastische, nicht militärische, den preußischen Wirtschafts- und Gewerbereformen verpflichtete Monument entstanden sein, der zivile wurde aber verbindlich mit dem monarchischmilitärischen Symbolort verkettet. Diesen Konnex sicherte der König einmal mehr dadurch, daß er nach der Feier zusammen mit dem Denkmalkomitee die

${ }^{42}$ Vgl. ibid. Zu Beuths Werdegang vgl. auch Wilhelm TREUE, Christian Peter Wilhelm Friedrich Beuth, in: Wolfgang TREUE, Karlfried GRU'NDER (Hg.), Berlinische Lebensbilder, Bd. 3: Wissenschaftspolitik in Berlin. Minister, Beamte, Ratgeber, Berlin 1987 (Einzelveröffentlichungen der Historischen Kommission zu Berlin, 60), S. 119-134.

43 Vgl. Aufruf des Central-Komite, GStA PK I. HA Rep. 93 C Nr. 2363, Bl. 46.

44 Die meisten Innungen stellten zehn Mann fur die Deputationen, Maurer, Zimmerleute und Weber als die von Beuth besonders geförderten Gewerbezweige durften mit je 50 Mann vertreten sein, dazu kamen knapp 100 Maschinenbauarbeiter. Vgl. Communal-Blatt der Haupt- und Residenzstadt Berlin, $\mathrm{N}^{\circ} 19$ vom 12. Mai 1861, S. 150.

45 Vgl. VZ, 14. Mai 1861, S. 2. 
Kommandantur verließ und »zu Fuß zu dem Platz des Denkmals« zurückkam, um es »umdrängt von der Volksmenge« zu begutachten ${ }^{46}$. Damit sicherte sich Wilhelm I. einen weiteren der bis dahin noch seltenen öffentlichen Auftritte als neuer preußischer Regent, demonstrierte "Volks«-Nähe und profitierte von den Loyalitätsbekundungen, die ihm seitens der Festversammlung entgegenschlugen. Darüber hinaus galten für die Festordnung die gängigen hierarchischen Staffelungsprinzipien ${ }^{47}$. Religiös-christliche Versatzstücke standen den dominant nationalstaatlich-preußischen deutlich nach, indem die Feier zwar mit einem Choral begann, das Denkmal aber mit den schwarz-weißen preußischen Fahnen umhüllt war und der Rede des preußischen Generalsteuerdirektors und Komiteevorsitzenden Johann von Pommer-Esche nach Hochrufen auf den König, die »Nationalhymne« folgte, bevor nach der eigentlichen Enthüllung und einer weiteren Ansprache des liberalen Handelsministers von der Heydt der Zug an Denkmal und König vorbei die Feier beschlo $\beta^{48}$.

Pommer-Esche zielte in seiner Ansprache auf das Exeptionelle einer Denkmalehrung für den "geräuschlosen« Förderer von Industrie und Handel. Hatten Monarchendenkmäler Kontinuität und Legitimität, Militärdenkmäler äußere (und innere) Wehrhaftigkeit und im tödlichen Opfergang bezeugte Sinnhaftigkeit der Nation beschworen, eröffnete die öffentliche Ehrung eines Protagonisten der Industrie neue Konnotationsfelder für die Nation, thematisierte ihre materielle Basis, ihre Ressourcen und Potentiale in Ausrichtung auf die $\mathrm{Zu}$ kunft und nahm außerhalb der Nation statt des »Feindes« eher das »Ausland» wahr, mit dem es zu konkurrieren galt. Der »vaterländische Gewerbefleiß [sollte] zum wetteifernden Kampfe mit dem Auslande (...) voranschreiten« und würde sich dabei auch auf »die eigene Kraft und [den] Trieb des Volkes« zu stützen haben ${ }^{49}$. Ähnlich wie im Rahmen öffentlicher Ehrung französischer Wissenschaftler in Paris wurde die Idee fortschrittlicher Ressourcenmobilisierung damit zugleich zum machtpolitischen Argument umgewandelt, geriet der internationale Konkurrenzkampf der ökonomisch potenten Nation in den Zielhorizont der Fortschritts-Apologeten. Pommer-Esche selbst unterbrach die Assoziation von eigener Dynamik und Zukunftgerichtetheit, indem er am Ende emeut die traditionale Wertschätzung der preußischen Monarchie einforderte, die durch die "Segnungen des Friedens" den »National-Reichthum« erst ermöglicht habe: »rüstiges Vorwärtsschreiten (...) aber auch (...) unverbrüchliche

46 Hierüber berichtete nur die NPKZ vom 14. Mai 1861, S. 2.

${ }^{47} \mathrm{Vgl}$. VZ, ibid. Auf einem abgegrenzten Raum vor dem Denkmal befanden sich Plätze für die geladenen Vertreter der städtischen und staatlichen Körperschaften (Abordnungen beider Kammern, der Ministerien, der Universität, der Akademie, der Gerichtsbehörden u.a.). Die Studierenden des Gewerbeinstituts und der Bauakademie, Handwerker und Innungen hatten sich hinter dem Denkmal aufzustellen, dahinter die übrigen Besucher mit Eintrittskarten und das "sonstige Publikum."

48 Vgl. ibid.

49 Vgl ibid. 
Treue und Hingebung gegen den König« blieben am Ende die Postulate, die mit dem Beuth- wie dem Thaer-Denkmal wzum Heil des Vaterlandes« aufgestellt worden waren ${ }^{50}$.

Beim der Feier folgenden Festessen im Krollschen Etablissement an der Westseite des Königsplatzes ${ }^{51}$ mit etwa 500 geladenen Gästen, darunter zahlreiche Staatsminister, die städtischen Gremien und Vertreter der von Beuth gegründeten Institutionen, bestimmten "Fahnen, Wappenschilder, Embleme des Handels, der Gewerbe, der Industrie und des Maschinenbaus« den Festschmuck und in der Mitte eine "mächtige allegorische Figur, die Göttin der Industrie ${ }^{52}$. Während damit Symbole selbstbewußter Eigenleistung und moderner Fortschrittsorientierung überwogen und auch die Tableaux vivants, die im Rahmen einer Theateraufführung gezeigt wurden, vor allem die »doppelt lebensgroße« Büste Beuths exponierten, wies zugleich ein "großes preußisches Wappenschild" zu Füßen der Allegorie auf die Zuordnung der Wirtschaftsbranchen und des individuellen Verdienstes zur preußischen Nation hin. "Heitere (...) Lied[er]«, zu denen nun aber keine Hymne zählte, der Abschluß der Feier mit einer "Sammlung« für Arme, und der Umstand, daß man nach dem Ende der Veranstaltung vin der heitersten und gemüthlichsten Stimmung (...) in traulichem Kreise « zusammenblieb ${ }^{53}$, zeugten sowohl von einem sozialen, sich zur paternalistischen Geste verstehenden Exklusivitätsbewußtsein der bürgerlichen Gäste als auch vom hohen Rang informeller Geselligkeit auBerhalb des offiziösen Programms.

Im Rahmen einer knappen Ansprache nutzte Rudolf Delbrück als Beuths Nachfolger an der Spitze des preußischen Gewerbevereins die Gelegenheit, auf dessen traditionelle Nähe zur Monarchie hinzuweisen, indem er auf die langjährige Mitgliedschaft des Monarchen im Verein verwies und Wilhelm I. im Jahr des Thronwechsels »in alter preußischer Treue« als dem neuen Regenten huldigte. Der Bezug auf die preußische und monarchische Nation diente hier nicht nur der Loyalitätsbekundung, sondern auch der vorauseilenden eigenmächtigen Sanktionierung der Festakte zum einen wie der Reformleistung in Handel und Gewerbe zum anderen ${ }^{54}$. Indem die abendliche Feier nur noch im sozialen Einzugsbereich des Bürgertums lag, wurde auf die für die Denkmaleinweihung noch kennzeichnende weite Inklusionsgeste vor allem nach unten nun verzichtet. Mit der Mischung auch Symbolen von Handel,

so Vgl. ibid.

51 Vgl. SCHÄCHE, Platz für die Macht, S. 34.

52 VZ, 14. Mai 1861, S. 2-4, hier S. 3.

53 Vgl. VZ, ibid. S. 4.

54 Weniger als Vertreter des Fortschritts gelangte 1869 auch der Architekt Karl Friedrich Schinkel zu einem Denkmal am Platz vor der Bauakademie und diente hier einem allgemeinen "Cultus der Kunst«; vgl. Subskriptionsaufruf des Schinkelkomitees vom 10. Juni 1858, BLHA Rep. 2A I P Nr. 718 [up]. 
Gewerbe und Industrie fand noch einmal eine separate, spezifisch bürgerliche Aneignung der Kultfigur statt, die mit einem entsprechenden Leistungs- und Selbstbewußtsein gefeiert wurde, dem die preußisch-nationalen Embleme beigeordnet blieben.

\section{London: Adaptionsprozesse eines neuen Deutungsmusters}

Auch in London gelangten, allerdings in noch bescheidenerer Zahl als in Paris und Berlin und mit äußerst mäßigem zeremoniellem Aufwand, Exponenten des Fortschritts zu Symbolmacht. Im Mai 1858 fand zunächst die feierliche Einweihung einer Bronzestatue für den Mediziner Edward Jenner am südwestlichen Ende von Trafalgar Square statt. Prince Albert selbst leitete die Zeremonie, würdigte den Philosophen und Philanthropen und bescheinigte ihm, wunestimable benefits (...) upon the human race« erworben zu haben ${ }^{55}$. Damit griff er nicht nur die Motivation des stiftenden Komitees auf, das den "great (...) benefactor of mankind « und Exponenten der »civilized world" ehren wollte $^{56}$. Zugleich setzte er sich darüber hinweg, daß die Denkmalerrichtung zum Gegenstand durchaus kritischer Anfragen im Unterhaus geworden war. Der Radikale Thomas S. Duncombe monierte dort die Standortwahl für das Monument »of this promulgator of cow-pock nonsense«, das sich auf Trafalgar Square höchst unpassend inmitten jenes Erinnerungsortes an die "naval and military heroes« Nelson, Napier und Havelock ${ }^{57}$ ausnehme $^{58}$. Dem Einwand lag nicht nur massive Skepsis an der Bedeutung von Jenners Verdiensten, sondern vor allem die Annahme zugrunde, daß die Aura des nationalen Symbolortes durch das nicht-militärische Leistungsprofil des Geehrten beeinträchtigt würde. Ein Votum ähnlicher Tendenz mündete schließlich in die grundsätzliche Anfrage nach den von der Regierung angewandten sfixed principles respecting the erection of statues in the metropolis « und in die Warnung vor einem unkoordinierten »disfigurement« im städtischen Zentrum ${ }^{59}$. Stellvertretend für die Regierung bekundete Lord John Manners in der Tat einen "general plan« zur symbolischen Gestaltung von Trafalgar Square, in dem die

Vgl. The Scotsman, 19. Mai 1858, PRO Work 20/33; knapp Daily Telegraph, 1. Mai 1858, S. 7.

56 Vgl. der Chairman des Komitees an das Office of Works vom 12. August 1857, PRO Work 20/33.

${ }^{57} \mathrm{Zu}$ Nelson's Column vgl. bereits Teil 1, Kapitel III.3.1.1. und zum 1856 errichteten Denkmal für Napier und zur Havelock-Statue von 1861 Kapitel III.3.2.

58 Vgl. Sitzung des HoC, 10. Mai 1858, Hansard $3^{\text {rd }}$ s. Bd. 150 (1858) S. 354.

59 Vgl. so der Peelite-Liberal Lord Elcho im HoC am 7. Mai 1858, ibid. S. 274-276. 
Jenner-Statue aber durchaus inbegriffen sein sollte ${ }^{60}$ und folglich nicht an die Einrichtung eines Denkmalforums für einen ausschließlich militärischen $\mathrm{Na}$ tionalismus gedacht war. Dennoch verfing die Kritik offenbar, denn bereits Anfang 1862 fand noch unter der Regierung Palmerston die zeremonielose Verlegung des Jenner-Denkmals an den nordöstlichen Rand der Kensington Gardens statt ${ }^{61}$.

Eine letzte, freilich am Ende erfolglose Initiative zu einem erneuten Standortwechsel des Jenner-Denkmals ging schließlich Ende 1896 von einem am Londoner St.George's Hospital konstituierten Komitee aus, das das international begangene Jubiläum zu Jenners ersten Impferfolgen zum Anlaß nahm, die erneute Verlegung der Statue in Hospitalnähe als der zentralen Wirkstätte des Mediziners zu beantragen. Damit sollte zugleich die anläßlich der Entfernung von Trafalgar Square demonstrierte Nachrangigkeit Jenners als nationaler Kultfigur kompensiert ${ }^{62}$ und der "general human and national concern « erneut als Qualifikation für die Denkmalwürdigkeit des Forschers an exponierter Stelle geltend gemacht werden ${ }^{63}$. So wenig ein erneuter Transfer der JennerStatue zustandekam, zeugten das späte Projekt wie bereits die Denkmalinitiative von 1858 doch vom gedanklichen Konzept einer Nation, die sich nicht nur äußerer Anfeindung kriegerisch zu erwehren hatte, sondern auch im Streben nach (medizinischem) Fortschritt mit andereren Nationen konkurrierte, um ihr Primat hier nicht durch Gewalt, sondern durch eine gleichsam moralische "great legacy to the world ${ }^{64}$ geltend zu machen.

Deutlich näher an der Thematik des Krieges als an der des medizinischen Fortschritts blieb demgegenüber eine Denkmalstiftung 1865 für Wellingtons renommierten Feldarzt während des Spanischen Unabhängigkeitskrieges und Gründer des National History Museums, Sir James McGrigor, auf dem Gelände des Chelsea Hospitals und damit an gleichsam halböffentlichem Ort. Die Initiatoren hatten sich bereits 1861 der Mühe unterzogen, die Statue nicht nur mit einschlägigen Urteilen Wellingtons selbst über den "most able and succesfull public servant he had ever met with « zu begründen und McGrigor damit direkt der Aura des nationalen Helden zu unterstellen ${ }^{65}$. Sie begründeten ihr Vorhaben zugleich mit der Ambition, nicht nur den militärischen Sieg, sondern

${ }^{60}$ Vgl. ibid.

${ }^{61}$ Vgl. Treasury vom 14. Juni 1861, PRO Work 20/33. Selbst in einem späten Memorandum über den Verbleib der Statue wurde der Transfer nur mit ästhetischen Bedenken des First Commisioners of Work begründet, der die Kombination von sitzender Jenner- und stehender Napier-Figur für ungeeignet erachtet habe. Vgl. Memorandum vom 20. November 1896, PRO Work 20/33.

62 Vgl. The Times, 1. April 1897, PRO ibid.

63 Vgl. The Times, 11. Dezember 1896, ibid.

64 Vgl. The Times, 1. April 1897, ibid.

${ }^{65}$ Brief des Denkmalkomitees an den First Commissioner of Works William Cowper, 5. Januar 1861, PRO Work 20/29 [up] 
auch die ihn ermöglichende Infrastruktur öffentlich zu honorieren und verarbeiteten hier nun die eindrückliche Erfahrung, die die britische Öffentlichkeit mit dem Bedarf an medizinischer Versorgung der Armee in den Jahren des Krimkrieges gemacht hatte. Als Gründer mehrerer »Benevolent Societies« schien McGrigor schließlich auch eine Nation zu repräsentieren, die nach dem Krieg die zivile Gesellschaft für Versorgungsleistungen gegenüber den Hinterbliebenen der Gefallenen in die Pflicht nahm und Unterstützungsangebote in der Logik einer Schicksalsgemeinschaft unterbreitete. Mobilisiert wurden schließlich auch Prestige- und Konkurrenzargumente: McGrigor sollte, so die Stifter, weitaus höher in der öffentlichen Wertschätzung rangieren als Napoleons Feldarzt Larrey, der nun aber doch in Paris schon Jahre zuvor geehrt worden war $^{66}$. Die Apostrophierung McGrigors als Vorreiter einer ebenso effizienten wie verläßlichen »nation« trug unter diesen Umständen nur allzu deutlich auch Züge einer offenen Symbolkonkurrenz und -defensive im Blick auf die französische Kapitale.

Schließlich entstand bis zum November 1866 am bereits von der Duke of York-Statue und dem Guards Memorial beherrschten Symbolort am Waterloo Place auf Initiative des Präsidenten der Royal Geographical Society, Sir R. Murchison und anderer "gentlemen ${ }^{67}$ eine Statue für den Expeditionsforscher Sir John Franklin. Seine frühe Karriere im Dienst der Navy, die Teilnahme an der Kanonade von Kopenhagen und mehrere Arktische Expeditionen verliehen ihm den Worten des First Lord of the Admiralty, Sir John Pakington, zufolge endgültig den Status des nationalen Heroen, dem mit Napoleon gemein war, in der Stunde des Sieges untergegangen zu sein. Solchermaßen war Franklin in das Pantheon jener "greatest and bravest of those naval heroes « aufgenommen «of whose glory and of whose memories England was so justly proud « ${ }^{68}$. Über aller festlichen Rhetorik blieb allerdings das Mißfallen der Denkmalstifter über die Verdrängung vom ursprünglich favorisierten Standort auf Trafalgar Square, die, wie Murchison anmerkte, in der Logik von Franklins Verdiensten auch im Rahmen der Schlachten von Kopenhagen und Trafalgar gelegen hätte ${ }^{69}$.

Allerdings stieß die Denkmalerrichtung auf äußerst mäßige Resonanz und erschien die Programmatik der Stifter kaum öffentlichkeitswirksam. Im Falle der Jenner-Statue verhinderten wissenschafts- und fortschrittskritische Ressentiments gegenüber der medizinischen Forscherleistung des Geehrten, im Falle McGrigors eine allzu enge Initiierung des Denkmals im Kreis der "Medical Officers of the Army«, daß ohnedies nurmehr vage assoziierte Nationsentwürfe klar vermittelbar schienen. Für die zeremonielle Zurückhaltung gab es allerdings durchaus zeitgenössische Deutungen. So gab die Times zwar

${ }^{66}$ Vgl. ibid. Zum Larrey-Denkmal vgl. bereits oben Kapitel IV.1.

${ }^{67} \mathrm{Vgl}$. Notiz der Treasury an das Office of Works vom 11. April 1863, PRO Work 20/85.

68 Vgl. The Times, 16. November 1866, S. 10.

69 Vgl. ibid. 
zu, daß auch die Franklin-Feier bescheiden blieb, beschloß aber, diesen Umstand als Indiz saturierten Nationalstolzes zu deuten: Ostentative Akte habe man in Großbritannien nicht nötig und leiste sich hier den »quiet, English way of doing things«, auch wenn andere Staaten eine vergleichbare Gelegenheit mit großem Pomp oder doch zumindest mit Flaggen, Musik und militärischen Symbolen inszeniert haben würden ${ }^{70}$.

\section{Vergleich}

Die vergleichsweise geringe Rolle der neuen Kategorie nationaler Kultfiguren, die mit den Repräsentanten von Reform und Fortschritt entstanden war, schlug sich am nachhaltigsten in London in einem gemessen an der Inszenierung anderer Personenkategorien massiven Zeremoniedefizit nieder. $\mathrm{Daß}$ die Pariser Larrey-Feier von 1850 sich vergleichsweise aufwendig ausnahm, war hier deutlich weniger der Fortschrittsthematik als ihrer spezifischen Verquickung mit Empire und Militär geschuldet. Die Festszene erschien hier einerseits unverkennbar militärisch dominiert, andererseits blieben Festdauer und Redebeiträge Ausnahmephänomene im Paris des Zweiten Empire. Vom militärisch geprägten Ritus und der sonstigen zeremoniellen Apathie Pariser Denkmalfeste hob sich das Prozedere der Berliner Denkmalfeste deutlich ab. Die Initiatoren des Thaer- wie des Beuth-Denkmals in Berlin 1860 und 1861 waren bemüht, dem Anspruch an eine nationale Feier mit einem hohen Maß an sozialer und regionaler und dabei dezidiert ziviler Inklusion zu genügen. Dabei gelang in beiden Fällen zumindest formal selbst die Integration von Arbeitern in die nationale Festgemeinde, die sich außerdem an den feierlichen Prozessionen beteiligten, während sowohl dieser Zug als auch die Verteilung der verschiedenen Gruppen über das Festterrain nach einer zuvor festgelegten Ordnung auf eine strikt berufsständische Gliederung der Formationen achtete. Ein auch nur annähernd vergleichbares $\mathrm{MaB}$ nicht nur an Aufwand, sondern auch an inklusiver, Traditionen bürgerlicher Festkultur aufgreifender Symbolik wiesen weder französische noch englische Inszenienungen zu ähnlich kategorisierbarem Personal auf. 1861 nutzte allerdings der nun anwesende, erst seit wenigen Monaten regierende Monarch den öffentlichen Auftritt, um die Bildregie der Feier mindestens ebenso sehr auf seine Person wie auf die Kultfigur zu zwingen und ließ sich im Rahmen des Festes gleichsam noch einmal akklamieren. Hymnen und Fahnen dienten in jedem Falle dazu, die Feierlichkeiten auch im Falle einer überregionalen Präsenz von Festteilnehmern definitiv auf die preußische

${ }^{70}$ Vgl. ibid. und The Morning Post, 16. November 1866, S. 5. 
Nation zu beziehen. Stärker als in Frankreich und England lebten im Zusammenhang mit dem Fortschritts-Personal Kultformen der bürgerlichen Feste in Berlin auch da lebhaft fort, wo die Einweihung des Beuth-Denkmals 1861 noch von einer abendlichen Feier ergänzt wurde, die zwar wieder von der Inklusionsgeste nach unten abrückte, aber nun mit bürgerlichem Eigenbewußtsein zur Selbst-Feier schritt, in der die preußische Staatssymbolik eher beigeordnet blieb. Hinter den Inszenierungen in Berlin blieben schließlich ähnlich wie die Pariser auch die Londoner Denkmalerrichtungen deutlich zurück. Die Einweihung der Jenner-Statue konnte auf dem Hintergrund vorausgegangener Querelen um ihre Plazierung kaum mehr eindrucksvoll wirken, die übrigen Denkmäler wurden in der Tagespresse kaum wahrgenommen. Die Deutung ritueller Apathie als Indiz saturierten Nationalstolzes blieb eine gewagte Selbstinterpretation nationaler Symbolinszenierungen seitens der Times.

In programmatischer Hinsicht gehörten in allen Fällen zur Feier der neuen Personenkategorie im öffentlichen Denkmal zunächst durchaus auch Phänomene strategischer nationaler Etikettierungen, die Denkmalkomitees vornahmen, um die öffentliche Relevanz ihres Vorhabens glaubhaft zu machen. Vor allem aber entstand mit den öffentlichen Monumenten für Reformer, Forscher und Mediziner in den europäischen Hauptstädten eine neue Kategorie national konnotierter Symbole. Reform und wissenschaftlicher Fortschritt wurden als neue Impulsgeber der Nation identifiziert, die nicht nur durch rückwärtsgewandte Bespiegelung ihrer Geschichte und gegenwartsbezogene Taxierung ihrer militärischen und politischen Ressourcen Kontur gewinnen, sondern über die Fortschrittsidee zugleich zukunftsorientiert und rational-pragmatisch auf ihre Effizienzsteigerung bedacht sein sollte. Die Nation-Fortschritt-Thematik blieb aber vor allem im deutschen und französischen Fall stark von den politischen Zusammenhängen der jeweiligen Systeme bestimmt. Das Pariser LarreyDenkmal gewann insofern eine besondere Dimension, als es 1850 bereits auf konservative Verhärtungen der republikanischen Ordnung hindeutete, indem ein Bezug auf republikanische Werthaltungen gänzlich fehlte, während von den Stiftern ein Deutungshorizont rückwärtig nach dem Ersten Empire hin entfaltet wurde. Deutungsmuster, die wenige Jahre später im Zusammenhang mit dem Denkmal für Marschall Ney Konjunktur haben sollten, wurden in der Larrey-Feier vorweggenommen, indem sich nun bereits die napoleonischen Kriege als loyalitätsprägender Faktor und als das zweite große Thema auch der Stiftung des Larrey-Denkmals erwiesen, während der Aspekt der Niederlage von 1815 allerdings noch ausgespart blieb. Mit dem Blick zurück auf das Empire verbanden die Initiatoren des Larrey-Monuments also nicht den ebenso rückwärtsgewandten Niederlagentopos, sondern zogen sich auf kompensatorische Deutungen zurück, indem sie sich an dem Nachweis abarbeiteten, daß in der Person des Mediziners Larrey auch eine vorwärtsorientierte, moderne Qualifikation der Nation Gestalt angenommen hatte, die nationales Selbstbe- 
wußtsein angesichts fortschrittlicher Errungenschaften erlaubte. Daß dann die konkrete militärische Leistungsbilanz der Nation nicht thematisiert werden mußte, wurde impliziert. Die explizite Verquickung mit dem Kriegsthema blieb demgegenüber streng selektiv, indem nicht der Zusammenbruch Frankreichs, sondern nur die Bewährung und Aufstockung französischer Militärressourcen in Form der von Larrey und seinen Helfern bereitgestellten medizinischen Versorgung der Soldaten angesprochen wurden. Nicht nur über die Zäsur von 1815 , sondern auch über die von 1830 und 1848 setzten sich die Redner 1850 schließlich hinweg, indem sie auf die systempolitisch indifferente Loyalität Larreys gegenüber dem »pays« abhoben. Die eindeutige Affinität der Larrey-Sympathisanten mit Napoleon deutete sich in entsprechenden Rekursen auf den Ersten Empereur gleichwohl unverkennbar an. Die Figur des Militärarztes an der Schnittstelle zwischen Krieg und Fortschritt avancierte auch in London mit dem McGrigor-Denkmal Mitte der 1860er Jahre zur Kultfigur und erschien hier spiegelbildlich zur Larrey-Napoleon I.-Konstellation an den Kult Wellingtons rückgebunden. Anders als in Frankreich konnte die Idee einer progressiven Infrastruktur hier aber mühelos dem Siegermythos von $1815 \mathrm{zu}-$ geordnet bleiben.

Die im Zweiten Empire noch folgenden Ehrungen für Forscherpersonal kaprizierten sich vor allem auf ein pragmatisches Effizienzargument. Ähnlich wie vor allem anläßlich der Feier des Berliner Beuth-Denkmals von 1861, spielte hier nun über den Gedanken wünschenswerter Produktivität der Nation hinaus bald die Idee der solchermaßen für den internationalen Konkurrenzkampf gerüsteten und nach mindestens ökonomischer "suprématie« strebenden Nation eine zunehmend wichtige Rolle. Der Reform- und Fortschrittsidee blieb damit im Spiegel der französischen wie der deutschen Denkmäler die Ambivalenz eigen, einerseits auf die materiellen Binnenverhältnisse der nationalen Ordnung zu sehen und die Mobilisierung von Ressourcen und Potentialen zunächst einmal als Wechsel auf eine erfolgreiche Zukunft zu verstehen, andererseits aber zugleich auf die Abgrenzung gegenüber andersnationalen Ordnungen mit dem Ziel eigener Überlegenheit abzuheben. Aggression oder Feindbildrhetoriken zählten indessen nicht zu den Versatzstücken dieser Deutung, weil der Konkurrent entweder nicht benannt, oder neutral als »Ausland« apostrophiert wurde.

Im Berliner Monument für Thaer als Exponent des agrarwirtschaftlichen Fortschritts spiegelte sich stärker als in Frankreich und ohnedies England wider, daß zum einen ungeachtet der steigenden Bedeutung der Industrie seit der Jahrhundertmitte die landwirtschaftliche Produktion in Preußen nicht anders als in den meisten deutschen Einzelstaaten dominant blieb ${ }^{71}$. Zum anderen schien sich unter den Bedingungen fehlender nationalstaatlicher Einheit der

${ }^{7}$ Vgl. WEHLER, Deutsche Gesellschaftsgeschichte, Bd. 3, S. 39. 
Verweis auf ökonomische Potenzen kompensatorisch zur Dokumentation nationaler Qualifikation besonders anzubieten. Der monumentale Rekurs auf die Nation verarbeitete also die landwirtschaftlich begründeten Prosperitätserfahrungen der Zeit und feierte den Repräsentanten agrarischer Reformen als Bürgen eines ebenso traditionellen wie flexiblen Wirtschaftssektors im Kraftzentrum der preußischen Nation. Mindestens ebenso stark wie dies für die französischen Denkmäler zu beobachten war, koppelten die Stifter des ThaerDenkmals den Exponenten des Fortschritts und die Idee der produktiven Nation unmittelbar an die monarchische Autorität zurück und mühten sich so ausdrücklich um die enge Verzahnung von Werttraditionen und innovativer Fortschrittsparole. Erst in öffentlichen Kommentaren deutete sich an, daß die Stilisierung einer produktiven, traditionalen Landwirtschaft zum materiellen und gleichsam gottgefälligen Rückhalt der preußischen Nation allmählich auch zugunsten anderer Erwerbszweige geweitet werden mußte. Mit der Monumentalisierung Beuths als Repräsentant von Handel und Gewerbe wurde dem vorsichtigen Einwand symbolisch Rechnung getragen und dann eine ähnliche Programmatik entfaltet. London blieb die einzige Stadt, in der sich die Weitung der Kategorie national konnotierter Kultfiguren gelegentlich auch auf dem Wege des Konflikts vollzog, indem die Initiatoren des Jenner-Denkmals auf mitunter harsche öffentliche Kritik bei ihrem Versuch stießen, die Statue auf Trafalgar Square zu plazieren, weil das Primat militärischer Qualifikation zum nationalen Heroentum für nicht erfüllt erachtet wurde. Die humanitäre und moralische »legacy« für die Nation, die die Stifter für das Monument geltend machten, litt unter den vorgetragenen Einwänden. Hinter dem Aufwand nicht nur an bürgerlicher Festkultur Berlins, sondern auch einer nachdrücklich vorgetragenen Programmatik jedenfalls blieben die Londoner Denkmäler deutlich zurück. 
